\title{
Le droit de la personne au Moyen-Orient
}

Baudouin DUPRET (Dir.), Standing Trial. Law and the Person in the Modern Middle East, New York, I.B. Tauris, 2004, 388 p.

Hélène Sallon

\section{OpenEdition}

\section{Journals}

Édition électronique

URL : http://journals.openedition.org/transcontinentales/1263

DOI : $10.4000 /$ transcontinentales. 1263

ISBN : 978-2-8218-1112-6

ISSN : $1775-397 X$

\section{Éditeur}

Editions de la maison des sciences de l'homme

Édition imprimée

Date de publication : 31 décembre 2006

Pagination : 148-150

ISBN : 978-2-200-92170-5

ISSN : 1950-1684

\section{Référence électronique}

Hélène Sallon, «Le droit de la personne au Moyen-Orient », Transcontinentales [En ligne], 3 | 2006,

document 11, mis en ligne le 12 octobre 2011, consulté le 25 septembre 2020. URL : http://

journals.openedition.org/transcontinentales/1263 ; DOI : https://doi.org/10.4000/transcontinentales. 1263

Ce document a été généré automatiquement le 25 septembre 2020

Tous droits réservés 


\title{
Le droit de la personne au Moyen- Orient
}

\author{
Baudouin DUPRET (Dir.), Standing Trial. Law and the Person in the \\ Modern Middle East, New York, I.B. Tauris, 2004, 388 p.
}

Hélène Sallon

\section{RÉFÉRENCE}

Baudouin DuPRET (Dir.), Standing Trial. Law and the Person in the Modern Middle East, New York, I.B. Tauris, 2004, 388 p.

1 Cet ouvrage collectif, dirigé par Baudouin Dupret, constitue l'une des premières études interdisciplinaires consacrée à la question de la personne dans les systèmes juridiques des sociétés arabes du xix́ siècle à nos jours. À partir de perspectives variées, les contributions questionnent « les relations entre l'individu, la société et les catégories structurelles des relations sociales » dans ce contexte, telles qu'elles s'expriment au travers du droit. Cette analyse fait l'hypothèse de l'existence dans ces sociétés d'un phénomène d'individuation qui se traduirait, au niveau juridique, par la place centrale accordée à la notion de personnalité juridique. Ce phénomène a souvent été décrit comme le résultat de l'importation de systèmes juridiques d'inspiration occidentale et, avec elle, de la transposition de dynamiques telles que la centralisation, l'individualisme et le sécularisme dans des sociétés de tradition islamique. L'interpénétration de conceptions différenciées de la personne dans le cadre juridique est ici explorée dans toute sa complexité par la combinaison de perspectives plurielles historique, juridique, sociologique, anthropologique et politique - et par le recours à une démarche pragmatique, qualifiée d'" approche praxéologique " par Baudouin Dupret. Cette approche vise à appréhender les perceptions du droit et de la personne dans les pratiques mêmes des acteurs afin de révéler « l'usage local, contextuel et contingent » de ces conceptions. 
2 La première partie de l'ouvrage pose les fondements épistémologiques et théoriques d'une réflexion sur la personne, le droit et leurs relations. Reprenant les analyses de Norbert Elias, Baudouin Dupret montre la nature contingente de la construction du concept de personne et de son imposition comme catégorie essentielle de l'organisation sociale et juridique des sociétés occidentales modernes. La personne, avec les concepts qui lui sont corrélés dans la philosophie occidentale, est ainsi devenue la pierre angulaire du droit libéral moderne. Cette conception du droit et de la personne s'est développée dans le contexte de l'individualisation de la société concomitante de la formation des États-nations et de dynamiques telles que la centralisation du pouvoir, la différenciation des fonctions sociales et leur interdépendance accrue, et l'institutionnalisation du contrôle des comportements. Mohamed Nachi analyse la construction de la catégorie de la personne dans la philosophie morale occidentale et islamique médiévale, et son usage dans une approche pragmatique des catégories du jugement et du sens de la justice. Cette catégorie, conçue comme entité morale articulant le «je » au " nous", permet d'appréhender le sens de la justice dans ses aspects individuels et collectifs, comme impératif de respect de la personne. Pour JeanNoël Ferrié, l'usage de cette catégorie de personne en sociologie est problématique en ce sens où elle ne rend pas compte de la dimension contextuelle des interactions et des perceptions déployées par les acteurs.

3 Les relations entre le droit et la personne dans le contexte des sociétés arabes sont abordées sous l'angle d'une part, des perceptions de la personne dans différents contextes juridiques et quasi juridiques et d'autre part, des définitions juridiques de la personne. Parmi les questionnements communs à ces contributions, l'on retrouve celui des modalités d'importation et de transplantation des conceptions morales et juridiques occidentales de la personne dans ces sociétés. Les contributions de Khaled Fahmy sur l'abolition de la torture en Égypte et d'Armando Salvatore sur la transformation normative de la Shari'a par les réformateurs musulmans à la fin du XIX ${ }^{e}$ siècle invitent à envisager les dynamiques et transformations locales qui ont amené le pouvoir et les élites à importer et à adapter des conceptions, techniques et institutions juridiques occidentales. De nombreux domaines du droit portent aujourd'hui la marque de ces importations juridiques qui ont remplacé ou suppléé les conceptions tirées de la Shari'a. C'est le cas du traitement juridique et judiciaire de la folie (Oussama Arabi), de l'application du principe de responsabilité individuelle et de la nature individuelle de la peine en droit égyptien (Murielle Paradelle) ou du développement de l'éthique médicale en Égypte (Baudouin Dupret). Le droit des minorités en Égypte constitue une exception (Maurits Berger), de par sa conception collective de la personne. Le juge y a privilégié l'impératif de maintien de l'ordre public, comme dans le domaine de la moralité. Cet impératif sous-tend une procédure islamique récemment utilisée en Égypte, la procédure en hisba, que Nathalie BernardMaugiron invite à envisager comme une forme locale de recours individuel au nom de l'intérêt collectif.

4 Ces contributions questionnent également la perception et la mobilisation de conceptions plurielles du droit et de la personne au travers des pratiques des acteurs dans des contextes juridiques ou quasi juridiques. Les pratiques sociales et juridiques des acteurs révèlent en effet des conceptions différenciées de la personne et une pluralité de systèmes normatifs. Le recours à des croyances traditionnelles, telles que la possession spirituelle (Barbara Drieskens), et à des modes de justice coutumière, tels 
que les comités d'arbitrage et le recours à la vengeance (Murielle Paradelle), traduisent la persistance de conceptions de la personne et de la justice concurrentes du droit positif en Égypte. Les acteurs mobilisent ces différentes perceptions de la personne et de la justice pour fonder leurs justifications dans le cadre d'une dispute commerciale entre vendeurs français et tunisiens (Mohamed Nachi). Dans le cadre de l'établissement de l'intentionnalité en droit criminel, les différents acteurs construisent et orientent leurs discours au regard des contraintes procédurales mais aussi de leur perception de la «normalité » (Baudouin Dupret).

5 L'approche privilégiée dans cet ouvrage permet ainsi de rendre compte de la complexité et de la pluralité des perceptions du droit et de la personne dans les sociétés arabes contemporaines, ce que n'aurait pas permis une analyse limitée aux textes et aux procédures juridiques. En replaçant l'étude de cette catégorie morale et juridique dans des dynamiques spatiales, sociales et politiques plus générales, cet ouvrage nourrit la réflexion sur de nombreuses thématiques à l'intersection de la société et du politique.

\section{AUTEURS}

HÉLĖNE SALLON

Centre de recherches français de Jérusalem (CRFJ) 